\title{
Comparação entre duas técnicas de biópsia renal guiadas por laparoscopia em eqüinos
}

\begin{abstract}
Alexandre de Faria TABET $^{1}$; Luís Cláudio Lopes Correia da SILVA ${ }^{1}$;

Idércio Luis SHINHORINI²; André Luis do Valle DE ZOPPA $^{1}$
\end{abstract}

\section{Correspondência para: LUÍS CLÁUDIO LOPES CORREIA DA SILVA \\ Departamento de Cirurgia \\ Faculdade de Medicina Veterinária e Zootecnia da USP \\ Avenida Prof. Orlando Marques de Paiva, 87 \\ Cidade Universitária Armando Salles de Oliveira \\ 05508-270 - São Paulo - SP \\ silvalc@usp.br}

Recebido para publicação: 24/07/2003 Aprovado para publicação: 15/03/2005

\author{
1- Departamento de Cirurgia da Faculdade de Medicina e Zootecnia da \\ USP, São Paulo -SP \\ 2- Departamento de Patologia da Faculdade de Medicina e Zootecnia da \\ USP, São Paulo -SP
}

\section{Resumo}

Palavras-chave: Eqüinos.

A biópsia renal é técnica de diagnóstico útil para identificação do tipo e severidade e acompanhamento da evolução da doença renal. A alta incidência de lesões renais em eqüinos, geralmente em conseqüência de distúrbios digestivos ou respiratórios, freqüentemente associados a endotoxemia, nos motivou a estudar a técnica de biópsia renal guiada por laparoscopia, comparando o uso de agulha com pinça de biópsia. Foram utilizados 10 eqüinos hígidos, distribuídos aleatoriamente em dois grupos. Em cinco animais foi realizada biópsia renal com agulha descartável do tipo tru-cut e nos outros cinco com pinça de biópsia de $5 \mathrm{~mm}$, sendo que o procedimento foi guiado em ambos os grupos com auxílio da laparoscopia. Os animais permaneceram em estação durante a biópsia, que foi realizada mediante sedação e analgesia e bloqueio anestésico local em 2 pontos no flanco esquerdo, sendo um para introdução do trocarte e laparoscópio e outro para introdução da agulha ou pinça. As técnicas foram comparadas quanto à segurança, intensidade do sangramentro, tempo de execução e significância da amostra colhida. Em todos os procedimentos, foi possível a inspeção da cavidade peritoneal e escolha do local de colheita do material, com ausência de acidentes ou complicações o que nos permitiu validar ambas as técnicas. A análise histológica dos fragmentos demonstrou maior porcentagem de biópsias consideradas adequadas no grupo em que foi utilizada pinça laparoscópica o que nos leva a indicar preferencialmente seu uso.

\section{Introdução}

A doença renal primária pode ser considerada rara em eqüinos, sendo geralmente conseqüente a distúrbios digestivos e respiratórios, freqüentemente associados à endotoxemia, como também causada pela nefrotoxicidade de alguns medicamentos administrados por tempo prolongado, em altas doses e associados à desidratação $\mathrm{o}^{1,2,3,4,5}$. Há de se considerar que, de acordo com Bayly et al. ${ }^{6}$, na maioria dos casos clínicos, o diagnóstico é feito baseado na detecção da azotemia, porém, somente quando $75 \%$ dos néfrons estiverem comprometidos será observado níveis de uréia e creatinina elevados. Isso vem ao encontro da observação feita por Brenner ${ }^{7}$, referindose à biópsia renal em seres humanos, onde $\mathrm{o}$ uso da técnica não somente aprimorou a sensibilidade diagnóstica, aumentando os conhecimentos referentes ao exame do sedimento urinário, como deu novas perspectivas ao estudo da patogenia das doenças renais.

A biópsia renal é procedimento comum em medicina humana e vem sendo utilizada de forma crescente em medicina veterinária como método auxiliar de diagnóstico nas doenças renais, principalmente em pequenos animais ${ }^{8,910}$. Em eqüinos, este procedimento 
foi citado por alguns autores ${ }^{11,12,13}$, podendose utilizar a laparoscopia como método complementar, tanto para identificação do ponto de colheita, como para inspeção da cavidade peritoneal. Em alguns casos, a biópsia renal é o meio diagnóstico indicado para definição de um prognóstico mais preciso e direcionamento do tratamento $0^{1,2,6,11,14,15}$.

Em eqüinos, durante a técnica de biópsia percutânea, a manobra de deslocamento manual do rim esquerdo feita pela palpação retal ${ }^{1,15}$, visando seu posicionamento mais próximo à parede abdominal esquerda, pode trazer desconforto ao paciente mesmo com o uso da ultrasonografia ${ }^{14}$, sendo relatadas pelos autores possíveis complicações, como hematomas subcapsulares, retroperitoneais, laceração renal e hemorragia da artéria e veia renal.Quanto à técnica de laparoscopia, possíveis intercorrências trans-operatórias são citadas $^{11,12,16}$ como laceração de vísceras quando da introdução de trocarte e desconforto dos animais causado pela pressão do pneumoperitônio.

Um dos fundamentos da laparoscopia é a infusão de gás no interior da cavidade abdominal, visando a obtenção de espaço para promover um adequado campo de visão pela óptica ${ }^{11,12,16,17,18,19,20}$. A introdução deste gás pode ser feita por uma agulha (Veress), catéter ou cânula mamária, antes da introdução do laparoscópio ${ }^{12,17}$. Uma vez inspecionada a cavidade, pode-se optar pelo local de introdução do segundo trocarte, por onde será passada a pinça de biópsia.

A biópsia renal é técnica útil para identificar o local de lesão, evolução e gravidade da doença renal. $\mathrm{O}$ intuito deste trabalho foi descrever detalhadamente a técnica com a utilização da laparoscopia como guia, comparando o uso da agulha tru-cut com a pinça de biópsia com relação ao tempo de procedimento, intensidade do sangramento, segurança e significância da amostra colhida.

\section{Materiais e Métodos}

Para a execução desse experimento foram utilizados 10 eqüinos hígidos; machos, pesando entre 350 e $450 \mathrm{Kg}$ e distribuidos aleatoriamente em dois grupos de cinco animais cada. No primeiro grupo, o instrumento utilizado para retirada da biópsia renal foi a agulha do tipo tru-cute no segundo grupo utilizou-se a pinça de biópsia laparoscópica. Em ambos os grupos, o procedimento foi feito em posição quadrupedal, utilizando-se do mesmo procedimento anestésico, bem como condutas pré e pós-operatórias idênticas. Precedendo o ato cirúrgico, já com os animais em tronco de contenção, foi realizada a palpação abdominal por via retal, para avaliação do posicionamento das alças intestinais, além de descartar a presença de possíveis aderências no ponto de inserção do primeiro trocarte.

Após tricotomia e anti-sepsia do flanco esquerdo foi administrado romifidina ${ }^{a}$ (Sedivet-Boehringer Ingelhein) na dose de $0.1 \mathrm{mg} / \mathrm{Kg}$ por via intravenosa e na seqüência foi realizado o bloqueio anestésico com $20 \mathrm{ml}$ de cloridrato de lidocaina $2 \%{ }^{\mathrm{b}}$ (Lidoston-Ariston), nos pontos de inserção dos trocartes. Decorridos 15 minutos, já com equipamento e o campo cirúrgico montados, foi administrado tartarato de butorfano ${ }^{c}$ (Torbugesic-Fort Dodge) na dose de 0,05 $\mathrm{mg} / \mathrm{Kg}$, também por via intravenosa.

Realizou-se incisão de pele com aproximadamente $1,5 \mathrm{~cm}$ feita na região central do flanco esquerdo, permitindo a introdução do primeiro trocarte ${ }^{\mathrm{d}}$ (Ethicon) [11 mm f] e colocação do laparoscópio ${ }^{\mathrm{e}}$ (Ótica de $10 \mathrm{~mm} 0^{\circ}$ - Asap), para em ato contínuo, já com visão da cavidade peritoneal, ser estabelecido o pneumoperitônio ${ }^{\mathrm{f}}$ (Insuflador de $\mathrm{CO}_{2}$ eletrônico, Argus) até atingir a pressão intraperitoneal controlada em 8 mmHg. A seguir, foi feita a segunda incisão de pele, dorso-cranialmente à primeira, a fim de permitir a introdução da agulha ou pinça de biópsia, como descrito a seguir:

\section{Grupo 1}

Foi feita incisão de pele de aproximadamente $0,5 \mathrm{~cm}$, seguida de 
introdução da agulha de biópsia ${ }^{g}$ (Tru-cut 20/16 semi-automática, Gallini) através da musculatura abdominal e peritônio. Já no interior da cavidade e uma vez transpassadas a fáscia perirrenal e cápsula renal, a agulha foi introduzida na porção caudo-lateral do parênquima renal, acionando-se o disparo automático. Após a retirada, avaliou-se a qualidade do fragmento conforme seu tamanho e aspecto e caso o mesmo parecesse inadequado, a punção se repetiu. Finalizando o procedimento, o pneumoperitônio foi desfeito e a pele suturada com fio de náilon $\mathrm{n}^{\circ} 2-0$ em pontos simples separados.

\section{Grupo 2}

A incisão de pele nesse grupo foi de aproximadamente $1 \mathrm{~cm}$ para passagem do segundo trocarte ${ }^{\mathrm{h}}$ (Ethicon) [5mm f], por onde foi introduzida tesoura laparoscópica para incisão da fáscia perirrenal em $1,0 \mathrm{~cm}$ de extensão, em direção vertical. Após a retirada da tesoura ${ }^{\mathrm{i}}$ (5 mm, Auto-suture), a pinça de biópsiaj $(5 \mathrm{~mm}$ oval fenestrada, Wisap) foi introduzida pelo mesmo trocarte para a extração de fragmento da porção caudo-lateral do rim. O mesmo procedimento de sutura descrito anteriormente foi utilizado para o grupo dois, após avaliação do fragmento e descompressão da cavidade peritoneal.

Após a biópsia, observou-se a intensidade do sangramento. Este foi classificado como intenso, quando a coleção de sangue alojou-se no espaço nefroesplênico em toda sua extensão; moderado, quando a coleção de sangue se alojou em parte do espaço nefro-esplênico e em pequena quantidade, quando observado apenas um filete de sangue saindo pelo orifício provocado no peritônio, próximo ao rim. Foi também avaliado o tempo de procedimento cirúrgico, bem como o acompanhamento clínico dos animais nos três dias anteriores aos procedimentos e nas três semanas subseqüentes, mensurando-se temperatura retal, tempo de preenchimento capilar, freqüência cardíaca, freqüência respiratória, além da avaliação da coloração de mucosas, apetite e freqüência de defecação e micção.

Os fragmentos de biópsia foram fixados em meio Bouin e transferidos para solução alcóolica $(70 \%)$, onde foram conservados para posterior processamento e leitura histológica em lâminas coradas com hematoxilina e eosina. Para análise, foi levado em conta a qualidade e grau de significância das amostras.

\section{Resultados}

A técnica anestésica empregada manteve os animais posicionados no tronco de contenção com ausência de movimentos indesejados, não havendo desconforto quando da introdução do instrumental ou infusão de gás na cavidade peritoneal.

Em dois dos animais submetidos à biópsia por agulha, foi observado aumento de volume no ponto de punção, imediatamente após a retirada da agulha, indicando a formação de hematoma retroperitoneal.

A intensidade do sangramento, imediatamente após a biópsia, está representada na tabela 1 .

O tempo médio de procedimento, incluindo inspeção geral da cavidade abdominal pelo flanco esquerdo e documentação fotográfica; gasto médio de $\mathrm{CO}_{2}$ por animal e a pressão média da cavidade peritoneal antes da instauração do pneumoperitônio estão apresentadas na tabela 2.

As dimensões dos fragmentos retirados pela agulha foram em média de 12 $\mathrm{mm}$ de comprimento, 1,5 $\mathrm{mm}$ de largura e $1 \mathrm{~mm}$ de espessura. Com a pinça laparoscópica, os mesmos possuíam em média $6 \mathrm{~mm}$ de comprimento, $6 \mathrm{~mm}$ de largura e $4 \mathrm{~mm}$ de espessura (Figura 1).

A análise histológica dos fragmentos foi considerada adequada em dois animais do grupo onde se utilizou a agulha e em quatro animais no grupo realizado com a pinça. Nas lâminas consideradas adequadas, observou-se número mínimo de cinco 
glomérulos, túbulos contornados e vasos, possibilitando a realização de diagnóstico histológico.

Os animais não apresentaram sinais de infecção ou desconforto no período pósoperatório, mesmo sem receber antibiótico, antiinflamatórios ou analgésicos posteriormente ao procedimento. Não foi observada diferença estatisticamente significativa nos valores de freqüência cardíaca, freqüência respiratória e temperatura retal. Não houve alteração de apetite, defecação, micção, coloração das mucosas ou tempo de preenchimento capilar durante as três semanas de avaliação pósoperatórias.

\section{Discussão}

Consideramos que a colocação dos animais em tronco de contenção, a realização de palpação retal previamente aos procedimentos, bem como o protocolo anestésico utilizado, colaboraram efetivamente para que o ato cirúrgico transcorresse de forma segura, sem acidentes relacionados à introdução dos trocartes ou durante a manipulação com o instrumental.

O uso do opióide associado ao sedativo, juntamente com o bloqueio anestésico local, mostrou-se satisfatório ${ }^{16,18,19}$, sendo discutível a necessidade de complementação com

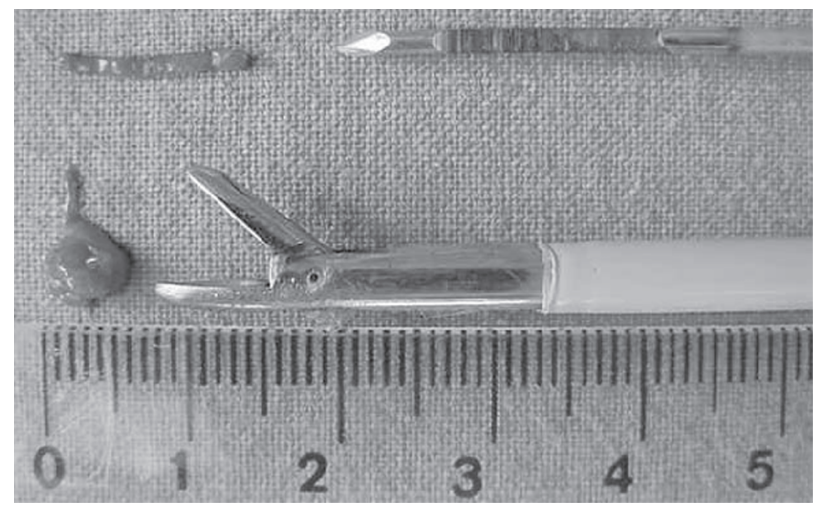

Figura 1 - Exemplos de fragmentos de biópsia colhidos pela agulha do tipo "Tru-cut" (acima) e pinça de biópsia laparoscópica (abaixo). São Paulo - 2002

Tabela 1 - Distribuição do número de animais por intensidade do sangramento no local da punção, avaliado após a biópsia, nos grupos agulha e pinça. São Paulo, 2002

\begin{tabular}{|l|c|c|}
\hline \multicolumn{1}{|c|}{ HEMORRAGIA } & AGULHA $\left(\mathrm{n}^{\circ}\right.$ de animais $)$ & PINÇA $\left(\mathrm{n}^{\circ}\right.$ de animais $)$ \\
\hline INTENSA & 2 & 1 \\
\hline MODERADA & 1 & 2 \\
\hline PEQUENA & 2 & 2 \\
\hline
\end{tabular}

Tabela 2 - Média do tempo de procedimento (minutos), consumo de $\mathrm{CO}_{2}$ (litros) e pressão intra-cavitária (mmHg) prévia, mensurados durante a biópsia renal nos grupos agulha e pinça. São Paulo - 2002

\begin{tabular}{|c|c|c|c|}
\hline GRUPOS & $\begin{array}{c}\text { TEMPO MÉDIO DE } \\
\text { PROCEDIMENTO }(\mathrm{min})\end{array}$ & $\begin{array}{c}\text { CONSUMO MÉDIO } \\
\mathrm{DE} \\
\mathrm{CO}_{2}(\mathrm{~L})\end{array}$ & $\begin{array}{c}\text { PRESSÃO } \\
\text { PRÉVIA MÉDIA } \\
(\mathrm{mmHg})\end{array}$ \\
\hline agulha & 20 & 28,9 & -5 \\
pinça & 35 & 35,9 & $-3,6$ \\
\hline
\end{tabular}

min - minutos; L - litros; mmHg - milímetros de mercúrio 
anestesia epidural ${ }^{12}$.

A quantidade de gás a ser infundida está na dependência do porte do animal, sendo que a pressão intracavitária ideal está relacionada à necessidade do procedimento ${ }^{19}$, porém, alguns autores ${ }^{12}$ não recomendaram pressão superior e $20 \mathrm{mmHg}$. Silva et al. ${ }^{19}$ utilizaram pressão entre 15 a $20 \mathrm{mmHg}$, obtendo adequada distensão da parede abdominal sem observar desconforto ou alterações respiratórias relacionadas ao pneumoperitônio. A opção pelo valor de 8 $\mathrm{mmHg}$ para realização da biópsia renal deveu-se à alta probabilidade de se trabalhar futuramente com animais debilitados hemodinamicamente, procurando interferir o mínimo possível com as condições do paciente, sem detrimento da qualidade do procedimento, corroborando com Silva et al. ${ }^{20}$ que mencionaram pressão de 06 a 08 $\mathrm{mmHg}$ em procedimentos de rotina.

A utilização da agulha de Veress, cânula mamária ou catéter ${ }^{12}$ para insuflação da cavidade abdominal não foi necessária no presente experimento, devido ao emprego de trocarte com sistema de segurança, o qual possui revestimento plástico da lâmina cortante que a recobre após a penetração do peritônio, evitando assim perfuração acidental de vísceras.

A visão do rim a ser biopsiado, possível pela laparoscopia ${ }^{13}$, forneceu alto grau de segurança aos procedimentos. A palpação retal foi desnecessária durante o procedimento, o que não comprometeu a eficácia da técnica, pelo contrário, poupou os animais do desconforto causado pela realização dessa manobra de forma profunda, indicada para manipulação do rim esquerdo quando outras técnicas são empregadas ${ }^{1,14,15}$.
Acreditamos que o motivo pelo qual se obteve melhor qualidade dos fragmentos retirados com a pinça foi a possibilidade de visão direta da cápsula renal, após secção e divulsão da fáscia e gordura perirrenal, fornecendo exata noção da profundidade de penetração da pinça para atingir o parênquima renal. Tal fato não foi possível com a agulha de biópsia, pois sem a divulsão perirrenal não se pode precisar a distância entre a fáscia e o parênquima renal. Uma possível variação na técnica, na ausência da pinça de biópsia, seria a divulsão da fáscia e gordura perirrenal para posterior introdução da agulha Tru-cut, com visão direta da cápsula renal. O formato da pinça de biópsia faz com que o fragmento seja retirado de uma porção mais externa do rim, diminuindo as chances de penetração em um grande vaso com sua utilização sem comprometer a representatividade da amostra. O tempo médio de procedimento com a pinça foi maior quando comparado ao da agulha, pela necessidade do uso da tesoura para divulsão da fáscia perirrenal.

A biópsia renal em eqüinos, realizada pela laparoscopia, com o animal em estação, demonstrou ser procedimento seguro e eficaz, permitindo visão do rim a ser biopsiado, além de avaliação da cavidade peritoneal. Com base nos resultados, recomendamos o uso de pinça, porém, não descartamos a possibilidade de utilização da agulha, podendo-se recomendar ambas as técnicas.

Sendo assim, esta técnica pode ser utilizada não apenas para determinação do tipo de lesão renal previamente reconhecida, mas também no acompanhamento de animais hemodinamicamente comprometidos, tanto na rotina clínica como em estudos experimentais.

\section{Comparison of two laparoscopic guided renal biopsy techniques in horses}

\section{Abstract}

Renal biopsy is a useful diagnostic technique for the identification to the type of lesion, severity of the disease and its evolution. The high incidence of renal lesions in horses, which usually are a
Key-words:

Equine.

Laparoscopy.

Kidney.

Biopsy. 
consequence of digestive and respiratory diseases and are frequently associated with endotoxemia, prompted us to study the laparoscopic technique for renal biopsy compair the use of needle and forceps. For the purpose of this study ten helthy horses were randomly chosen and divided into two grups of five animals each: in one group laparoscopic guided renal biops was performed using trucut needles and in the other group using $5 \mathrm{~mm}$ laparoscopic forceps. Procedures were carried out with the animals in standing position. Animals were given a sedative analgesic combination and local anesthetic was infiltrated in two specific points on the left flank. One point was used to introduce the trocar and laparoscopic, the other was used to introduce the forceps or the needle. The techniques were compared as regards safety, intensity of bleeding, time for execution and significance of the sample obtained. In all of the procedures, were possible to inspect the abdominal cavity, and chose the place to collect samples, without acidents or complications. The histological analysis of the sample showed a higher percentage of adequate biopsies in the group where laparoscopic forceps were used that permit us to viable both of them with preference for the forcps use.

\section{Referências}

1 BAYLY, W. M.; PARADIS, M. R.; REED, S. M. Equine renal biopsy: Indications, technic, interpretation and complications. Modern Veterinary Practice. v. 61, n. 9, p. $763-768,1980$.

2 BAYLY, W. M. Insuficiência Renal Aguda. In: REED, S. M.; BAYLY, W. M. Medicina interna eqüina. Rio de Janeiro: Guanabara Koogan, 2000. p. 727-734.

3 CUNILLERAS, E. J.; HINCHCLIFF, K. W. Renal Pharmacology. Veterinary Clinics of North America. Equine Practice, v. 15, n. 3, p. 647-664, 1999.

4 DIVERS, T. J. et al. A. Equine glomerulonephritis and renal failure associate with complexes of group-c streptoccocal antigen and IgG antibody. Veterinary Immunology and Immunopathology, v. 32 p. 93-102, 1992.

5 DIVERS, T. J. et al. Acute renal failure in six horses resulting from haemodynamic causes. Equine Veterinary Journal, v. 19, n. 3, p. 178-184, 1987.

6 BAYLY, W. M. et al. A reproducible means of studying acute renal failure in the horse. Cornell Veterinary, v. 76, p. 287-298, 1986.

7 BRENNER, B. M. Brenner and rector's the kidney. 5. ed. Philadelphia: W.B. Saunders, 1996.

8 GRAUER, G. F.; TWEDT, D. C.; MEROK. N. Evaluation of laparoscopy for obtaining renal biopsy specimens from dogs and cats. Journal of American Veterinary Medical Association, v. 183, n. 6, p. 677-679, 1983.

9 JERAJ, K.; OSBORNE, C. A.; STEVENS, J. B. Evaluation on renal biopsy in dogs and cats. Journal of American Veterinarian Medical Association, v. 181, n. 4, p. 367-
369,1982

10 WACKES, J. Laparoskopische und thorakoskopische biopsieentnahmen bei hund und katze. Kleintierpraxis. v. 41, n. 6, p. 411-418, 1996.

11 FISCHER Jr., A. T. Standing laparoscopic surgery. Veterinary Clinics of North America. Equine Practice, v. 7, n. 3, p. 641-647, 1991.

12 HENDRICKSON, D. A.; WILSON, D. G. Instrumentation and techniques for laparoscopic and thoracoscopic surgery in the horse. Veterinary Clinics of North America. Equine Practice, v. 12, n. 2, p. 235251, 1996.

13 TROSTLE, S. Gastrointestinal Endoscopic Surgery. Veterinary Clinics of North América. Equine Pratice. v. 16, n. 2, p. 329-341, 2000.

14 MODRANSKY, P. D. Ultrasound-guided renal and hepatic biopsy techniques Veterinary Clinics of North America. Equine Practice, v. 2, n. 1, p. 115-126, 1986.

15 OSBORNE, C. A. et al. Percutaneous renal biopsy in the cow and horse. Journal of American Veterinarian Medical Association, v. 153, n. 5, p. 563-570, 1968.

16 GALUPPO, L. D.; SNYDER, J. R.; PASCOE, J. R. Laparoscopic anatomy of the equine abdomen. American Journal of Veterinary Research, v. 56, n. 4, p. 518-531, 1995.

17 HENDRICKSON, D. A. Endoscopic surgery. Veterinary Clinics of North America: Equine Practice, v. 16, n. 2, p. 233-388, 2000.

18 RAGLE, C. A.; SCHNEIDER, R. K.; SOUTHWOOD, L. L. Abdominal laparoscopy in horses. Compendium 
on Continuing Education for the Practicing Veterinarian/ Equine, v. 18, n. 11, p. 1231-1239, 1996.

19 SILVA, L. C. L. C. Emprego da laparoscopia na colheita de biópsia hepática em eqüino: estudo da técnica e avaliação pós-operatória. 1998. 88 f. Tese (Doutorado em Cirurgia Veterinária) - Faculdade de Medicina Veterinária e Zootecnia, Universidade de São Paulo, São Paulo, 1998.

20 SILVA, L. C. L. C. et al. Aplicação clínica da laparoscopia em eqüinos. Revista de Educação Continuada do CRMV-SP, v. 3, n. 3, p. 12-20, 2000. 